\title{
Image Classification with Shell Texture Feature Extraction Using Local Binary Pattern (LBP) Method
}

\section{Putri Aisyiyah Rakhma Devi ${ }^{*}$ dan Rizqi Putri Nourma Budiarti}

\author{
${ }^{1}$ Teknik Informatika, Fakultas Teknik Universitas Muhammadiyah Gresik, Gresik \\ ${ }^{2}$ Information System, Engineering Department, Universitas Nahdlatul Ulama Surabaya, Surabaya \\ *Corresponding Author \\ E-mail: deviaisyiyah@umg.ac.id*
}

\begin{abstract}
Classification procedure that is usually done manually by way of separation based on the texture of the shell shell. Classification is done by looking at objects based on inherent characteristics usually referred to as features / characteristics. Classification by hand can cause accuracy problems. In the image of the shells, texture characteristics are needed to distinguish one type of shell from another. The purpose of this study is to develop a texture feature extraction system for the classification of shell images. The input image is carried out preprocessing and segmenting to separate objects from the background and the image of the separated object is transformed into a grayscale image for the feature extraction process using the Local Binary Pattern method. Based on trials that have been done, the accuracy is quite good, the highest accuracy value occurs in shellfish blood cockles with RBF kernels. While the lowest accuracy is on testing the feather shell image where the accuracy value is $86.6 \%$ this result can show that the LBP method with SVM classification is quite reliable in calculating the accuracy for the classification process of shellfish types.
\end{abstract}

Keywords: texture feature extraction, LBP, classification, shell image.

\section{INTRODUCTION}

Indonesia is one of the countries that has the largest area of marine waters in the world. Geographically, the waters of Indonesia are located in tropical regions which are rich in various natural marine resources. Utilization of marine resources is not only done through capture, but also needs to be developed cultivation business. The existing fisheries sector is still exploring sea products, namely tuna, shrimp and seaweed, while various types of mollusks are not yet desirable to develop. One type of mollusk is a shell which is an abundant fishery product in the tropics and a good and inexpensive source of animal protein for the community [1]. Shellfish and foods from the sea are rich in amino acids and fatty acids. Shellfish can also be developed into one of the reliable export products. Therefore, making shells as an interesting object to be studied. Shellfish are marine animals that belong to a group of soft-bodied animals oyster family, flat and layered, and has a pair of shells that are connected by hinges so that it can be opened and closed. Each type of shell has a different shell texture and shape. Each shell of the shell has a characteristic pattern on the texture and shape of the shell. It is this style and shape that distinguishes one shell from another one[2]. Automatic separation (classification) can be done by utilizing computer technology, especially in image processing and for the classification can be solved using Support Vector Machine (SVM)[3][4]. One important part that can support the success of the classification process is the pattern recognition process. Pattern recognition process is carried out by extracting 
features from existing patterns. The characteristic that is used to recognize the pattern of shells is the texture characteristics. Texture characteristics can describe the arrangement of intensities of a set of pixels that are next to an image. Therefore, the exact texture feature extraction method is needed in order to achieve high accuracy results in the classification process.

One method for extracting good texture features is Local Binary Pattern (LBP). LBP is a simple but quite efficient method in representing texture characteristics[5]. LBP is a gray-scale invariant or unaffected method for uneven lighting in an image, because LBP describes the texture locally to support the local contrast measure from an image[6]. In addition, LBP only consists of a few neighboring pixels with uncomplicated operations. Local Binary Pattern (LBP) is a texture analysis method that uses statistical and structural models. LBP analyzes the texture locally in the spatial domain, by comparing the intensity of the pixels between the central pixels and the neighboring pixels at a certain radius. So that gradient information can be obtained to represent edges, points, and other local features of an image. Next, a histogram is arranged to determine the distribution of the gradient values. With this simple calculation method, making LBP quite reliable on images that have different lighting [7].

Local Binary Pattern (LBP) is a texture analysis method that uses statistical and structural models. Many researcher using LBP for pedestrian detection[8], medical image analysis[9], car detection[10], license plate number detection[11], music genre classification[12] and facial expression recognition[13] but others researcher also using it for texture feature extraction and classification such as Jie Sima et all [14] applied extended Contrast LBP by using the extracted feature including sign feature, energy feature, and the center pixel feature by constructing the histogram based in the feature of the sign energy center pixel before and also combine it with chisquare distance and the nearest neighbor classifier to perform texture classification. . LBP analyzes the texture locally in the spatial domain, by comparing the intensity of the pixels between the central pixels and the neighboring pixels at a certain radius. So that gradient information can be obtained to represent edges, points, and other local features of an image. With this simple calculation method, making LBP quite reliable on images that have different lighting[5]. LBP steps in extracting features are:

1. For each pixel, the LBP value is calculated by comparing the intensity of the pixel between the intensity of the central pixel with the intensity of its neighboring pixels at a certain radius, as shown in Figure 1. The intensity of the central pixel becomes the thresholding to arrange the LBP value in each pixel of the image.

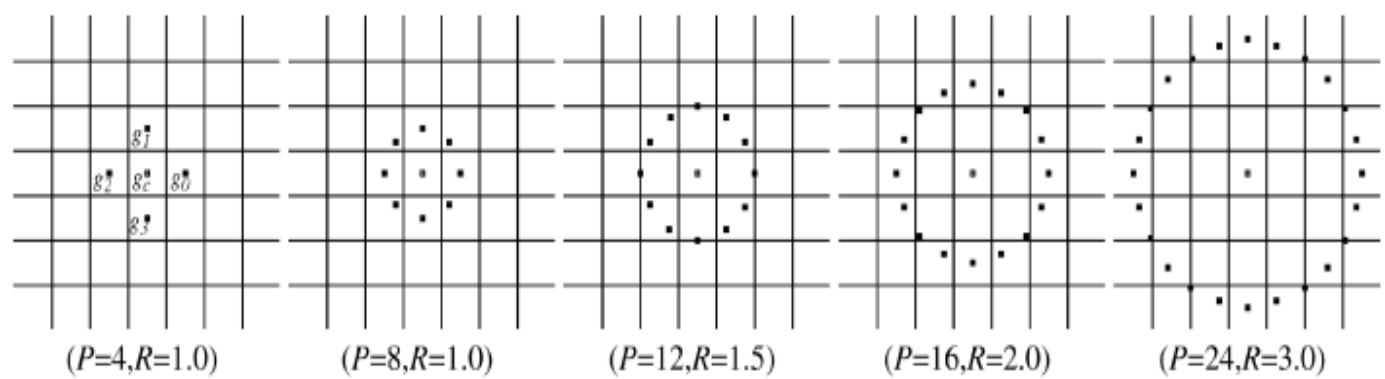

Figure 1. Local Binary Pattern Operator

2. If the value of the intensity of the central pixel is greater than the value of the intensity of the neighboring pixels, the binary transformation value for the central pixel is one. Conversely, if the value of the intensity of the central pixel is smaller than the value of 
the intensity of the neighboring pixel, the binary transformation value for the central pixel is zero, as shown in Figure 2 (b).

3. The binary values of the neighboring pixels are arranged, as shown in Figure 2 (c)

4. The arrangement of the binary value is converted to a decimal value, by multiplying the binary value by its weight, as shown in Figure 2 (c) and Figure 2 (d).

5. Mathematically, LBP calculations can be written based on Equation (1).

$$
L B P_{P, R}=\sum_{p=0}^{P-1} s\left(I_{p, R}-I_{c}\right) 2^{P}
$$

Where,

$$
s(x)= \begin{cases}1, & x \geq 0 \\ 0, & x<0\end{cases}
$$

$P$ is the number of neighbors, $R$ is the radius between the center and neighbor, $L B P_{P, R}$ is the decimal value of the conversion binary value, $I_{C}$ is the intensity value of the central pixel, $I_{p, R}$ is the intensity value of neighboring pixels $p$

$(p=0,1, \ldots,(P-1))$ with radius $R$. Whereas $s(x)$ is a thresholding function.

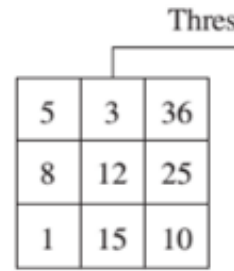

(a)

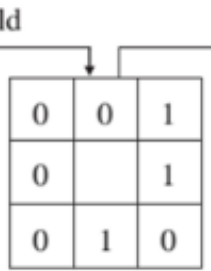

(b)

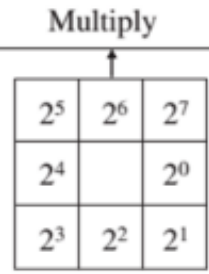

(c)

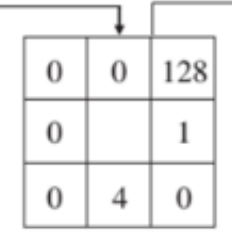

(d)

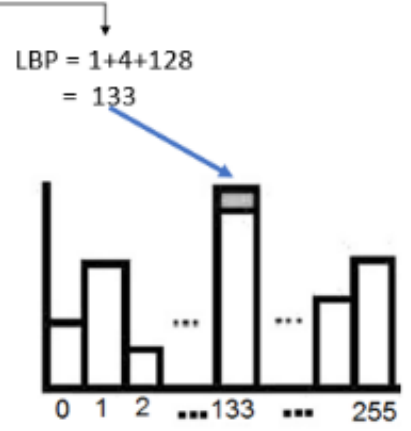

(e)

Figure 2. Illustration of LBP Process

(a) Original image. (b) The result of comparison with the thresholding function. (c) Weight. (d)

Results of multiplication with weights. (e) Histogram of LBP features.

6. A histogram is arranged, as shown in Figure 2.5 (e). Mathematically, a histogram can be defined by Equation (2.3).

$$
H_{j}=\sum_{x, y}^{L-1} \begin{gathered}
I(x, y)=j, \\
j=0,1, \ldots, n-1
\end{gathered}
$$

$H_{j}$ is the histogram value at the $j$ intensity, $I(x, y)$ is the intensity value at the pixel coordinates (x,y), $L$ is the measure of the number of pixels in an image, and $\mathrm{n}$ is the maximum value of intensity. 


\section{RESEARCH METHODS}

The design of this study, there are several things that need to be considered, this research explained how to extract shell texture characteristics on the image of a shell using the Local Binary Pattern (LBP) method. Broadly speaking, the flow of research can be observed in Figure 3.

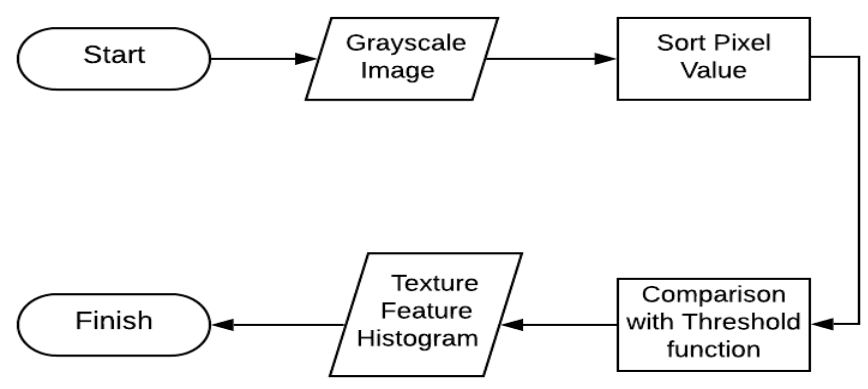

Figure 3. Extract Shell Texture Using Local Binary Pattern

At the analysis and design stage of an algorithm, an image before undergoing further processing needs to be done a preprocessing stage, which is a technique used to prepare an image in order to produce the desired output. The image that has gone through the preprocessing stage, then will go through the segmentation stage, namely the separation between the foreground and the object. In this study, the original image through the preprocessing stages is changing the original image in the form of an RGB image, then image processing is done by changing the RGB image into a grayscale image and then the canny edge, dilation and median filtering process is performed. The stages of the process are to get the shape of the edge of the shell (binary image) which will then be processed when extracting the shape features.

For the next stage, grayscale image through canny edge detection is used to get the edge of the object. The process of dilation and erosion is also used at this stage, then a skeleton process is used to represent the binary image of the object shell. In Figure 2. you can see the preprocessing and segmentation stages for each feature.

In the next stage, the implementation of algorithms against the designs that have been designed previously. The specifications of the hardware and software used consist of an Intel Core i3 $2.20 \mathrm{GHz}$ processor, $2.00 \mathrm{~GB}$ of memory capacity and a hard disk with a capacity of $500 \mathrm{~GB}$. As for the software specifications used in the implementation of this research algorithm, namely Microsoft Excel and MATLAB R2013a by utilizing existing libraries. 


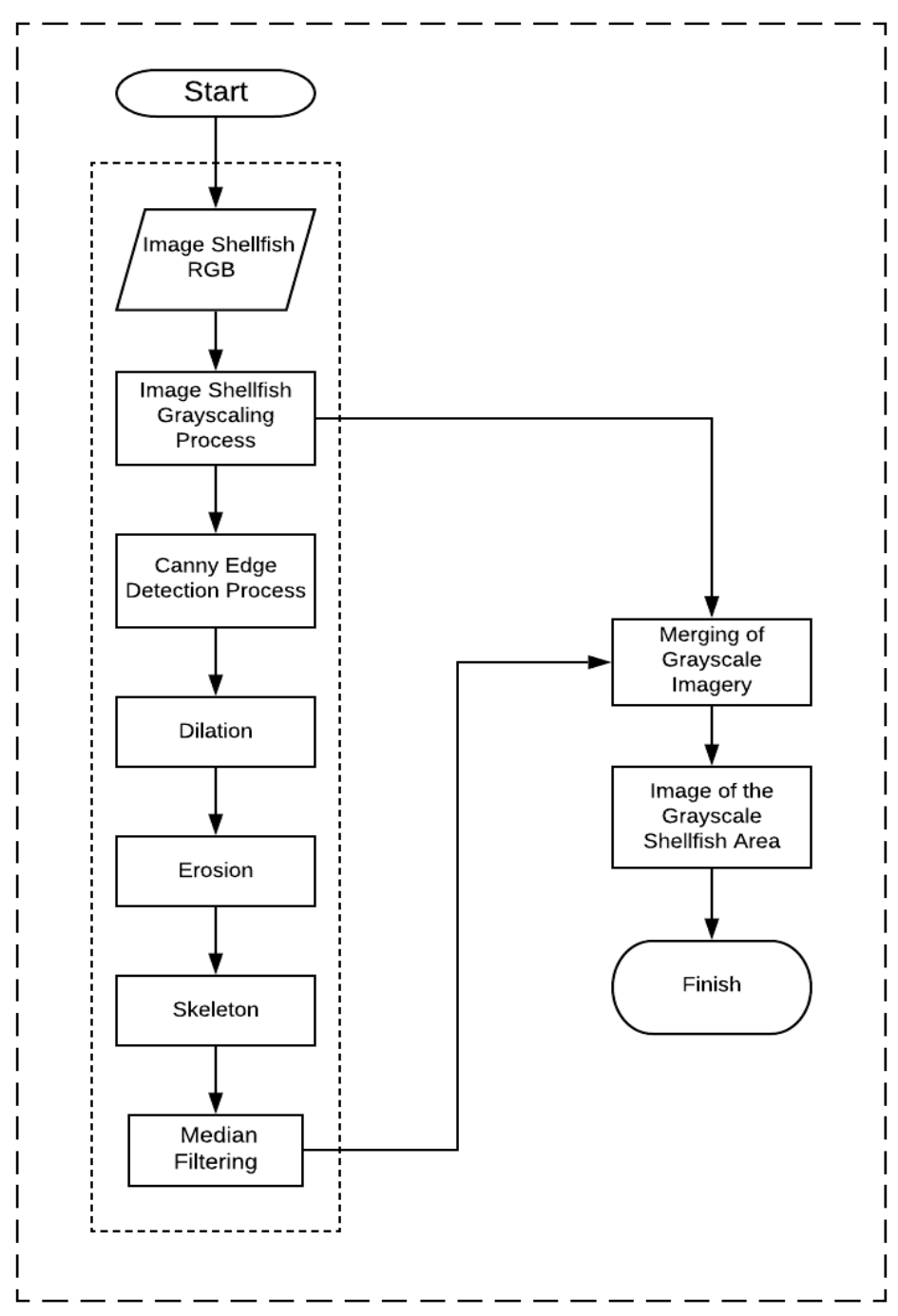

Figure 4. Pre-Process and Segmentation Stages

In the final stage to analyze data from the texture feature test results, it is expected that from the usury test analysis results will be obtained in accordance with the initial research objectives. For the process at the classification stage is done by using Support Vector Machine (SVM)[3][4]. Although in the beginning SVM was a classification for two classes, in its development SVM did not only classify two classes, but more than two classes (multiclass). The reason for using this classifier is because of its ability to generalize, its relatively easy implementation, and its ability to handle high-dimensional data. In the classification stage there are several kernel experiments performed namely linear kernel, Radial Basis Function (RBF) / Gaussian kernel and Polynomial kernel [3][4][15].

\section{RESEARCH RESULT AND DISCUSSION}

In this study the dataset used is a $300 \times 225$ RGB image. The number of datasets used as trial data is 60 images of clams consisting of 3 different classes / species, namely 20 images of blood shells, 20 images of sand shells, and 20 images of feather shells. Data collection was taken personally by researchers in the morning with good lighting. The shells used as a dataset are fresh consumption shells. An example of the dataset used is shown in Table 1. 
Applied Technology and Computing Science Journal, Vol. 3, No. 1, June 2020.

Table 1. Dataset Sample Shellfish Description

\begin{tabular}{ccc}
\hline No & Class & Images \\
\hline 1. & Shellfish Blood Cockles & \\
\hline 2. & Shellfish Sand Cockles & \\
& \\
\hline 3. & Shellfish fur Cockles \\
\hline
\end{tabular}

In Figure 3. It is a preprocessing stage for obtaining the texture of the shells. Initially a conversion of an RGB image (a) into a grayscale image (b) was followed by the merging of a grayscale image (c) and a segmented binary image to get the object area of the shell (d). The final result of this stage of the proposal is the formation of an output image which will become the input image at the feature texture extraction stage by the LBP method.

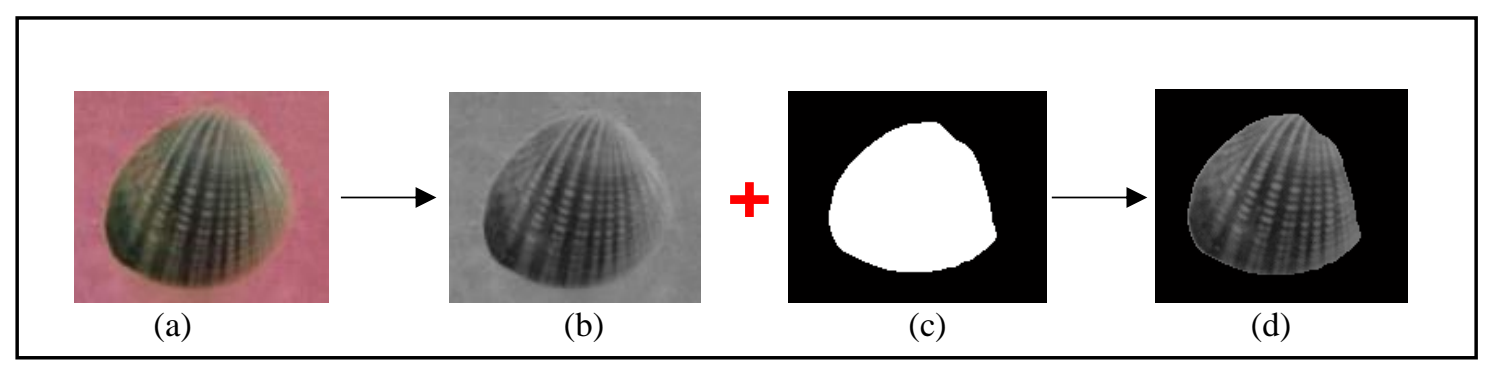

Figure 5. Preprocessing step to get the image of the texture of the shells

Then a test is performed on the feature testing using the LBP method. Get an average texture value with 256 texture histogram and 128 features. 


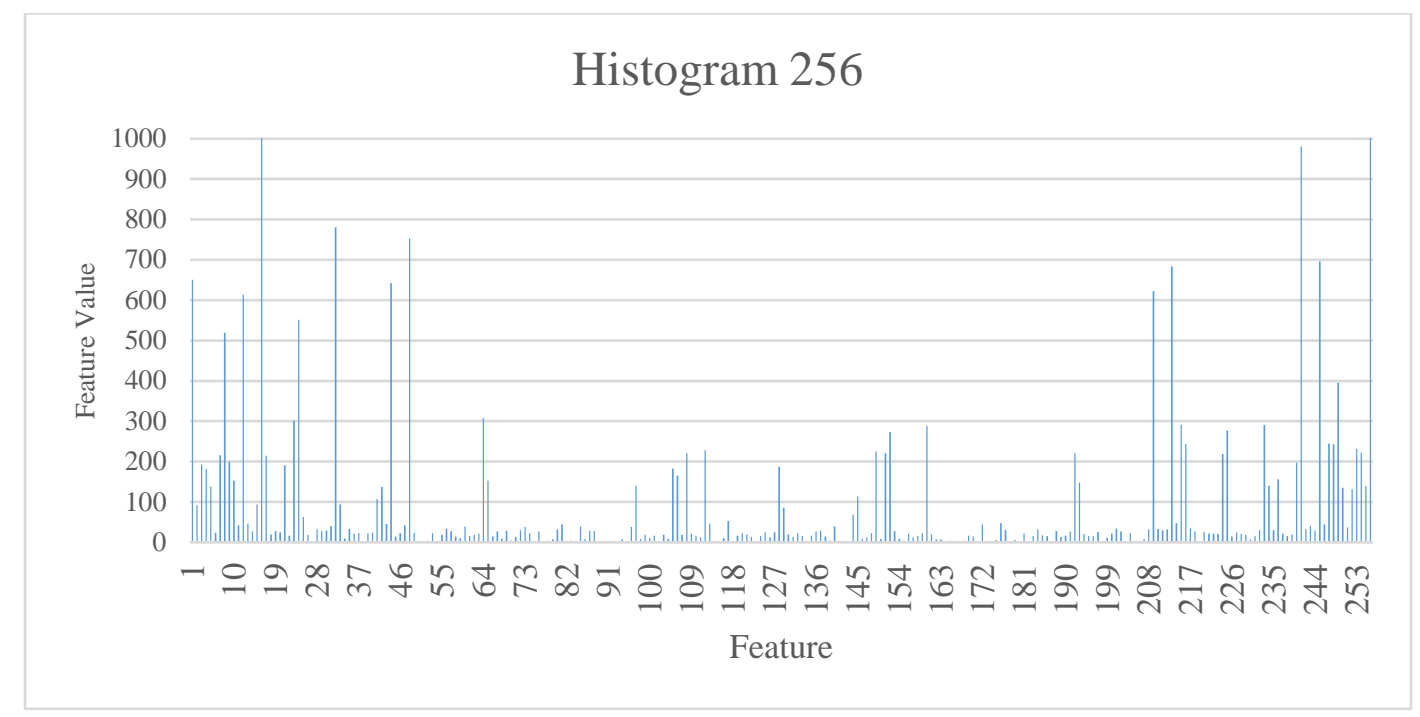

Figure 6. Histogram 256 Texture Characteristics

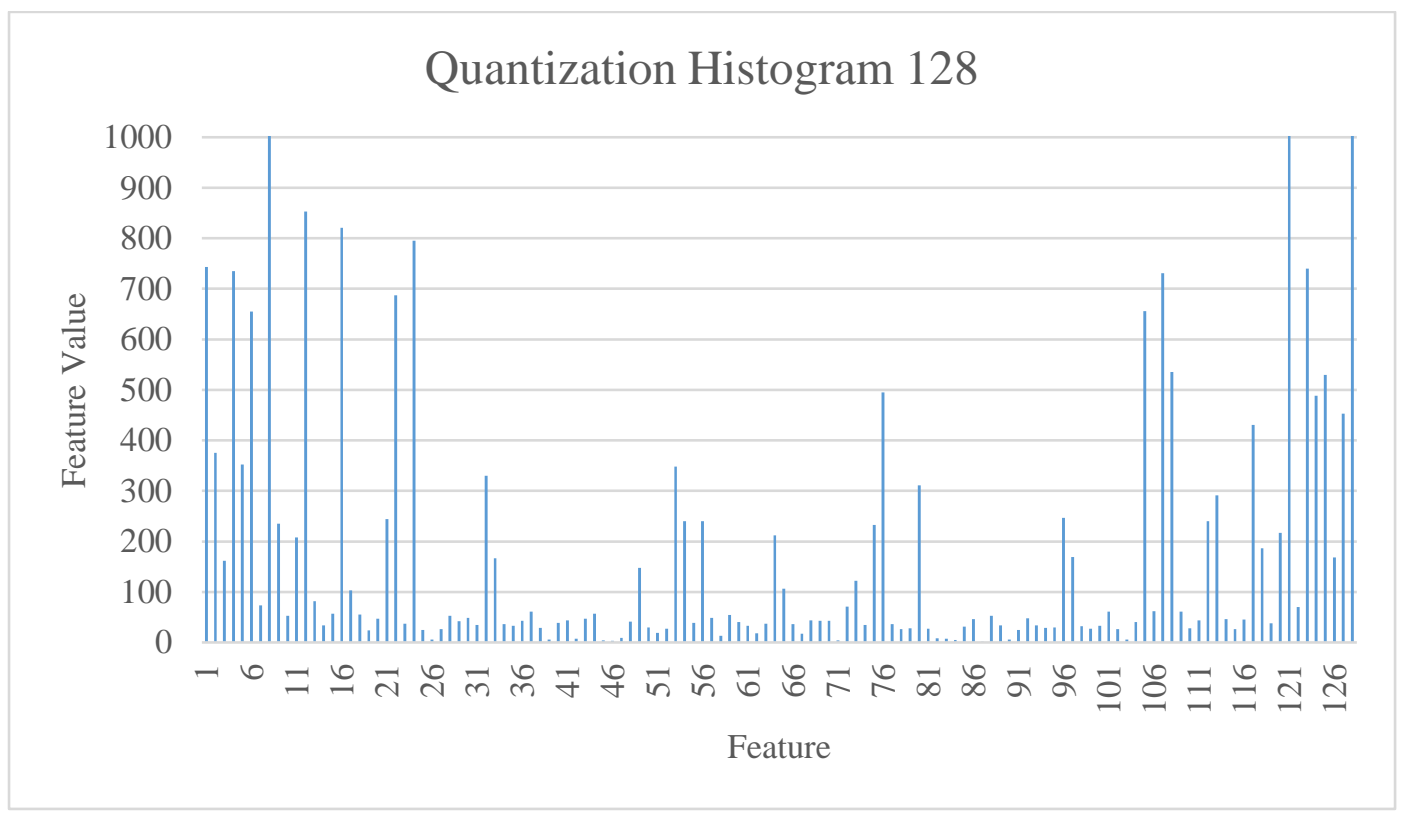

Figure 7. Texture Histogram 128 Characteristics

Next to test the texture feature classification results are performed with a quantization histogram along 128 and 256 features of the image of the shells in the existing dataset. In this research, we also classify using SVM, this research is carried out using linear SVM and the use of linear kernel and RBF kernel using parameter C. The C parameter in SVM is used to control the tradeoff between hyperplane margins and errors in classification. However, in this study, the parameter values of $\mathrm{C}=1$ and gamma $=0.1$ were used. the use of functions in $\mathrm{C}$ and gamma to determine the validation value of accuracy where the performance measurement uses the percentage of classification accuracy. Accuracy can be written as a comparison between the correctly classified image and the whole image being tested. The accuracy calculation is calculated as follows:

Accuracy $=($ Number of test images identified as correct / number of images tested $) \times 100 \%$ 
The parameters used in this experiment for linear kernels with parameter $\mathrm{C}=1$, while for $\mathrm{RBF}$ kernels the with $\mathrm{C}=1$ and gamma $=0.1$. Model testing of training data is testing the previously trained images and stored in a model where the training data used is 3 shells and each shell uses 20 images. The results of model testing on training data can be seen in the following Table 1 .

Table 2. Result of Training Data

\begin{tabular}{|l|l|r|}
\hline Kernel & Parameter Kernel & $\begin{array}{l}\text { Accuracy of } \\
\text { training data }\end{array}$ \\
\hline Linear & $\mathrm{C}=1$ & $100 \%$ \\
\hline RBF & $\mathrm{C}=1, \mathrm{\gamma}=0.1$ & $100 \%$ \\
\hline
\end{tabular}

Model Testing against Test Data. Image testing aims to find shells that have not been recognized or classified. The classification model used to test the image of the shell that has not been recognized uses the best model from the validation results and training data testing. The test data used consisted of 3 shells with 10 images of each shell.

Table 3. Result of Training Data

\begin{tabular}{|l|r|l|r|}
\hline Kernel & $\begin{array}{l}\text { Number image } \\
\text { test }\end{array}$ & $\begin{array}{l}\text { The correct } \\
\text { image } \\
\text { recognition }\end{array}$ & $\begin{array}{l}\text { Accuracy of } \\
\text { training data }\end{array}$ \\
\hline Linear & 30 & 28 & $93.30 \%$ \\
\hline RBF & 30 & 28 & $93.30 \%$ \\
\hline
\end{tabular}

In the results of research conducted with the success rate of introduction of shells with different variations in virgin, sand and feather shells, a graph of the accuracy of the recognition success rate was obtained.

Table 4. Result Accuracy of Shellfish Image Testing

\begin{tabular}{|l|r|r|}
\hline & Linear Kernel & \multicolumn{1}{l|}{ RBF Kernel } \\
\hline Shelfish Blood & $93.30 \%$ & $100.00 \%$ \\
\hline Shellfish Sand & $90.00 \%$ & $93.30 \%$ \\
\hline Shellfish Fur & $86.60 \%$ & $86.60 \%$ \\
\hline
\end{tabular}


Where in the virgin shell test, the highest value was obtained where $93.3 \%$ was for linear kernels while $100 \%$ was for RBF kernels. In the sand shells, the values obtained were $90 \%$ for the linear kernel and $93.3 \%$ for the RBF kernel. Whereas for the feather shells, the value was $86.6 \%$ in the linear kernel or the RBF kernel.

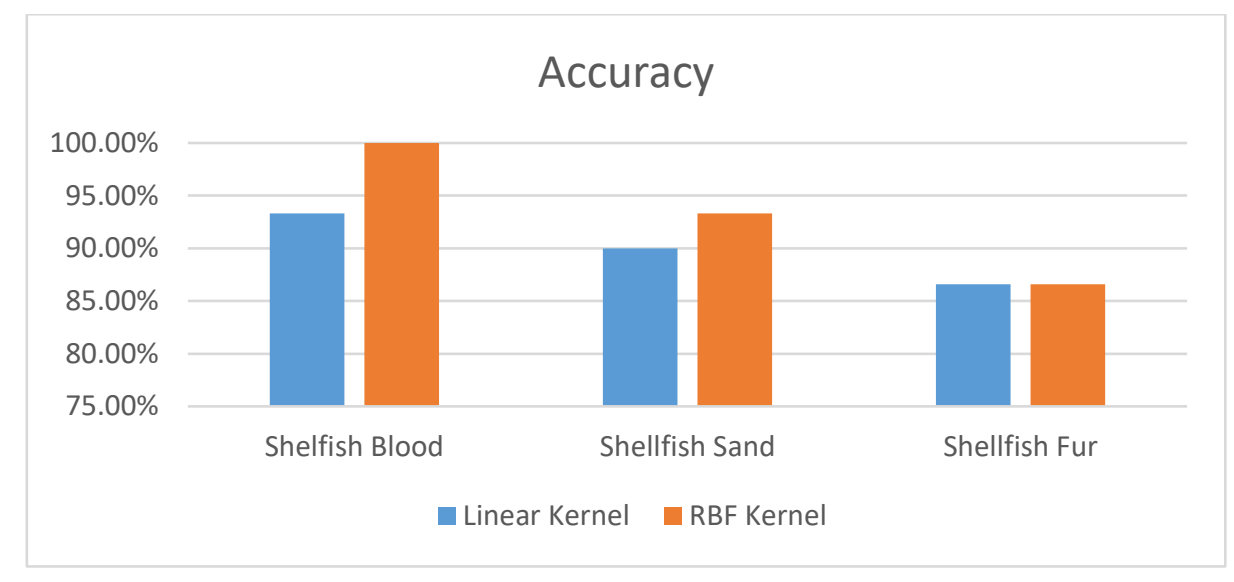

Figure 8. Accuracy of Shellfish Image Testing.

\section{CONCLUSION}

The results of the LBP (Local Binary Pattern) method are used to extract texture characteristics on the image of the shells obtained from the texture of the shells of the shells. The accuracy of classification obtained by using the SVM method gets a value that based on the results of the tests that have been carried out, the shellfish recognition system using LBP feature extraction has worked well on the use of $\mathrm{C}=1$ and gamma $=0.1$ where the accuracy value for testing and training data gives $100 \%$ results and the accuracy value on testing test data is $93,3 \%$. Meanwhile, the highest accuracy value occurs in virgin shells with RBF kernels. While the lowest accuracy is on testing the feather shell image where the accuracy value is $86.6 \%$ this result can show that the LBP method is quite reliable in calculating the accuracy for the classification process of shellfish types.

\section{REFERENCES}

[1] R. Hudaya, "Pengaruh Pemberian Belimbing wuluh (Averrhoa bilimbi) Terhadap Kadar Kadmium (Cd) Pada Kerang (Bivalvia) Yang Berasal Dari Laut Belawan Tahun 2010," 2010.

[2] N. Afiati, "Karakteristik Pertumbuhan Alometri Cangkang Kerang Darah Anadara Indica (L.)(Bivalvia: Arcidae)," J. Saintek Perikan., vol. 1, no. 2, pp. 45-52, 2005.

[3] A. S. Nugroho, A. B. Witarto, and D. Handoko, "Application of support vector machine in bioinformatics," in Proceeding of Indonesian Scientific Meeting in Central Japan, 2003, p. 2003.

[4] R. P. N. Budiarti, S. Sukaridhoto, M. Hariadi, and M. H. Purnomo, "Big Data Technologies using SVM (Case Study: Surface Water Classification on Regional Water Utility Company in Surabaya)," in 2019 International Conference on Computer Science, Information Technology, and Electrical Engineering (ICOMITEE), 2019, pp. 94-101.

[5] T. Ojala, M. Pietikainen, and T. Maenpaa, "Multiresolution gray-scale and rotation invariant texture classification with local binary patterns," IEEE Trans. Pattern Anal. Mach. Intell., vol. 24, no. 7, pp. 971-987, 2002.

[6] S. Ke-Chen, Y. A. N. Yun-Hui, C. Wen-Hui, and X. Zhang, "Research and perspective on local binary pattern," Acta Autom. Sin., vol. 39, no. 6, pp. 730-744, 2013. 
[7] M. Pietikäinen, A. Hadid, G. Zhao, and T. Ahonen, Computer vision using local binary patterns, vol. 40. Springer Science \& Business Media, 2011.

[8] Y. Cao, S. Pranata, and H. Nishimura, "Local binary pattern features for pedestrian detection at night/dark environment," in 2011 18th IEEE International Conference on Image Processing, 2011, pp. 2053-2056.

[9] L. Nanni, A. Lumini, and S. Brahnam, "Local binary patterns variants as texture descriptors for medical image analysis," Artif. Intell. Med., vol. 49, no. 2, pp. 117-125, 2010.

[10] S. Jabri, M. Saidallah, A. E. B. El Alaoui, and A. El Fergougui, "Moving Vehicle Detection Using Haar-like, LBP and a Machine Learning Adaboost Algorithm," in 2018 IEEE International Conference on Image Processing, Applications and Systems (IPAS), 2018, pp. $121-124$.

[11] B.-G. Han, J. T. Lee, K.-T. Lim, and Y. Chung, "Real-Time License Plate Detection in HighResolution Videos Using Fastest Available Cascade Classifier and Core Patterns," Etri J., vol. 37, no. 2, pp. 251-261, 2015.

[12] Y. M. G. Costa, L. S. Oliveira, A. L. Koerich, F. Gouyon, and J. G. Martins, "Music genre classification using LBP textural features," Signal Processing, vol. 92, no. 11, pp. 27232737, 2012.

[13] D. Huang, C. Shan, M. Ardabilian, Y. Wang, and L. Chen, "Local binary patterns and its application to facial image analysis: a survey," IEEE Trans. Syst. Man, Cybern. Part C (Applications Rev., vol. 41, no. 6, pp. 765-781, 2011.

[14] J. Sima, Y. Dong, T. Wang, L. Zheng, and J. Pu, "Extended contrast local binary pattern for texture classification," Int. J. New Technol. Res., vol. 4, no. 3, 2018.

[15] H. Maruta, A. Nakamura, and F. Kurokawa, "A new approach for smoke detection with texture analysis and support vector machine," in 2010 IEEE International Symposium on Industrial Electronics, 2010, pp. 1550-1555. 